\title{
The major CD8 T cell effector memory subset in the normal and Chlamydia trachomatis-infected human endocervix is low in perforin
}

Joyce A Ibana' ${ }^{1}$ Leann Myers ${ }^{3}$, Constance Porretta ${ }^{2}$, Maria Lewis ${ }^{1}$, Stephanie N Taylor ${ }^{4}$, David H Martin ${ }^{4}$ and Alison J Quayle 1* $^{*}$

\begin{abstract}
Background: The local tissue microenvironment plays an important role in the induction, homing, maintenance and development of effector functions of T cells. Thus, site-specific differences in phenotypes of mucosal and systemic T cell populations have been observed. Chlamydia trachomatis most commonly infects the endocervix in women, yet little is known about Chlamydia-specific effector T cell immunity at this unique mucosal site. Our previous flow-cytometry-based study of cervical-cytobrush retrieved cells indicated that CD8 T cells are significantly increased in the C. trachomatis-infected human endocervix. The cytolytic function of CD8 T cells is important in the protective immunity against many intracellular pathogens, and requires the cytolytic granule perforin to facilitate the entry of other molecules that mediate the lysis of target cells. Determination of perforin expression of the CD8 T cell population in the endocervix would therefore provide insights on the granule-mediated cytolytic potential of these cells at this site.
\end{abstract}

Results: Our histological data revealed that C. trachomatis-infected tissues have significantly higher numbers of CD3 and CD8 T cells compared to non-infected tissues $\left(p<0.01\right.$ ), and that the majority of $C D 8^{+}$cells do not express perforin in situ. A subsequent flow cytometric analysis of paired blood and endocervix-derived cells $(n=16)$ revealed that while all the CD8 T cell subsets: naïve, effector memory $\left(T_{E M}\right)$, central memory $\left(T_{C M}\right)$ and terminally differentiated effector memory $\left(T_{E M R A}\right)$ can be found in the blood, the endocervix is populated mainly by the $T_{E M}$ CD8 $T$ cell subset. Our data also showed that perforin expression in the $T_{E M}$ population is significantly lower in the endocervix than in the blood of $C$. trachomatis positive women $(n=15 ; p<0.0001)$, as well as in $C$. trachomatisnegative individuals ( $n=6 ; p<0.05$ ). Interestingly, our in vitro co-culture study suggests that the exposure of HeLa 229 cervical epithelial cells to IFN gamma could potentially induce a decrease in perforin content in CD8 TEM cells in the same microenvironment.

Conclusions: The low perforin content of CD8 TEM cells in the endocervix, the local site of C. trachomatis infection in women, may reflect the unique immunological environment that balances immune protection against sexually transmitted infections and immune- tolerance to support conception.

\footnotetext{
* Correspondence: aquayl@|suhsc.edu

${ }^{1}$ Microbiology, Immunology and Parasitology Department, Louisiana State University Health Sciences Center, New Orleans, Louisiana 70112, USA Full list of author information is available at the end of the article
} 


\section{Background}

CD8 $\mathrm{T}$ cells are key cellular components in the control of many intracellular microbial infections via their cytolytic function. Chlamydia trachomatis serovars D-K are intracellular bacteria that infect the columnar epithelial cells of the genital tract. Epithelial cells can present antigens in the context of MHC class I and activate a CD8 $\mathrm{T}$ cell immune response. Thus, investigation of the CD8 $\mathrm{T}$ cell cytolytic response to $C$. trachomatis infection is important as it could reveal a mechanism by which the bacterium is deprived of its intracellular niche. The major CD8 T cell cytolytic pathway involves the perforin and granzyme mediated induction of apoptosis $[1,2]$. Perforin mediates the delivery of granzymes to target cells by homopolymerization in the plasma membrane in a $\mathrm{Ca}^{2+}$ dependent manner producing pores that acts as a channel for granzyme entry $[3,4]$. Perforin is suggested to be necessary in CD8 $\mathrm{T}$ cell cytolytic activity, as perforin deficient mice have reduced efficiency in controlling viral infection [5].

During the course of infection, CD8 T cells differentiate and this is accompanied by changes in the expression of surface markers and functional capacity [6]. Naïve T cells are activated when they encounter their specific peptide-MHC complexes on professional antigen presenting cells [7]. A memory $\mathrm{T}$ cell differentiation pathway has been established by the group of Sallusto and Lanzavecchia, and others, whereby subsequent to antigen encounter, $\mathrm{T}$ cells proliferate and undergo phenotypic changes that modify their tissue homing properties [8-10]. Antigen-specific cells possessing a naïve-like phenotype $\left(\mathrm{CD} 45 \mathrm{RA}^{+} \mathrm{CCR} 7^{+}\right)$are recruited into a prememory subset before reaching the central memory $\left(\mathrm{T}_{\mathrm{CM}}\right)$ and effector memory $\left(\mathrm{T}_{\mathrm{EM}}\right)$ stages that are characterized as $\mathrm{CD} 45 \mathrm{RA}^{-} \mathrm{CCR} 7^{+}$and $\mathrm{CD}^{+} 5 \mathrm{RA}^{-} \mathrm{CCR} 7^{-}$respectively. Eventually, these memory $\mathrm{T}$ cells reach a terminally differentiated effector stage $\left(\mathrm{T}_{\mathrm{EMRA}}\right)$ characterized as CD45RA ${ }^{+}$CCR7 $^{-}$. Progression of a $\mathrm{T}$ cell through these subsets is associated with the acquisition of effector function and loss of proliferative potential [ibid]. Effector CD8 $\mathrm{T}$ cells acquire the capacity to migrate to extra lymphoid sites to sites of infection, and deliver perforin and granzyme at the immunological synapse to kill infected target cells [11]. CD8 T cell differentiation in response to infection is thus characterized by the acquisition of immunological properties that allow them to successfully clear intracellular pathogens.

Evidence that specific tissue microenvironments significantly influence CD8 T cell phenotype and function is accumulating. For example, Masopust et al., illustrated that the gastrointestinal microenvironment promotes differentiation of a unique memory CD8 $\mathrm{T}$ cell population, and that CD8 T cells can switch phenotypes with changes in anatomic location [12]. The influence of the anatomic micromilieu was also illustrated by a study by Shacklett et al., who demonstrated that unlike those in peripheral blood, CD8 T cells resident in the gastrointestinal tract of normal macaques were low in perforin. The absence of perforin in normal GI tissue was interpreted to be a mechanism by which the anatomical integrity of this mucosal site is protected, though it provides advantages for pathogens [13].

The female genital tract (FGT), like the GI tract, is also frequently exposed to foreign antigens, including commensal and pathogenic microorganisms. Furthermore, as a reproductive site, the FGT must tolerate foreign antigens to support conception. We thus hypothesized that CD8 T cells in the endocervix may also have limited cytolytic potential compared to their peripheral counterparts. Therefore, to investigate the phenotype of CD8 T cells in the FGT in the absence and presence of C. trachomatis infection, we sampled endocervical CD8 T cells from women to characterize the immune cell population, and assess the cytolytic potential of CD8 T cells at this site. In addition, using an in vitro approach, we further tested whether the presence of IFN gamma in a microenvironment could influence the perforin expression of CD8 $\mathrm{T}_{\mathrm{EM}}$ cells.

\section{Results}

CD8 T cells infiltrate the human endocervix during $C$. trachomatis infection

Consistent with our previous findings with cytobrushretrieved endocervical $\mathrm{T}$ cells, immunohistological staining for CD3 and CD8 T cell infiltrates in six C. trachomatisnegative and four $C$. trachomatis-positive banked endocervical tissues demonstrated that the number of $\mathrm{CD}^{+}$ and $\mathrm{CD}^{+} \mathrm{T}$ cells is significantly higher in C. trachomatispositive than in $C$. trachomatis-negative endocervical tissues ( $\mathrm{p}<0.01$ for both comparisons) (Figure 1). Interestingly, when we stained serial sections of endocervical tissues for CD8 and perforin, we observed that the majority of CD8 positive cells were perforin negative (Figure 2). Quantitative analysis of perforin-expressing CD8 T cells, however, was limited by the immunohistological single staining technique's inability to differentiate these cells from other immune cells that also express perforin, such as eosinophils, basophils, mast cells and NK cells. Thus, while our immunohistochemical data provided significant insights on the make-up of T cell infiltrates and suggested that CD8 T cells expressed limited amounts of perforin, we recognized that a multiparameter flow cytometric analysis would be needed to support and extend these observations.

\section{The CD8 T cell repertoire in the human endocervix is distinct from the periphery}

To further analyze the endocervical immune cell repertoire, we performed multiparameter flow cytometric 


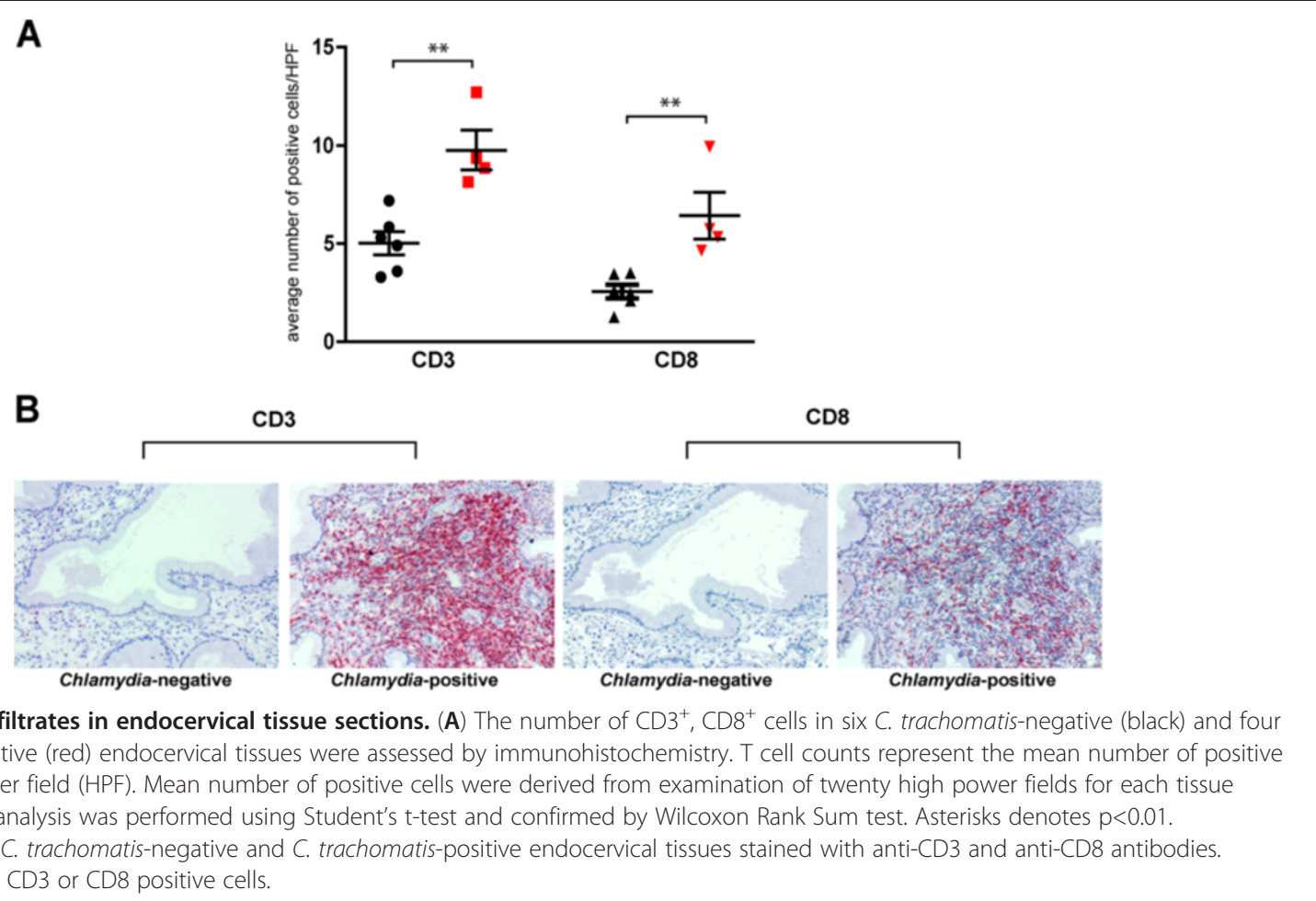

analyses to determine the mononuclear leukocyte types in isolated peripheral blood mononuclear cells (PBMC) and cytobrush-retrieved endocervical cells from $15 \mathrm{C}$. trachomatis-infected and 6 uninfected young women attending the Delgado Clinic. The gating strategy utilized in this study is shown in a representative analysis flow chart shown in Figure 3. Utilizing this strategy, we identified the lymphocyte population based on forward (FSC) and side-scatter (SSC) profile, from which the NK cell, CD4 T cell, and CD8 T cell subpopulations were delineated. We then assessed the perforin and granzyme expression in each of these cell types from paired samples obtained from the blood and endocervix. NK cells and $\mathrm{CD} 4 \mathrm{~T}$ cells were utilized as cellular controls, while granzyme B was utilized as an intracellular staining control. Perforin and granzyme B expression of the different immune cells from the blood and endocervix of a representative $C$. trachomatis-negative and C. trachomatis-positive women is shown in Figure 4. Consistent with previous reports, we observed that perforin expression is relatively low in CD4 T cells, and high in NK cells [14,15].

We then further delineated the CD8 $\mathrm{T}$ cell population into memory subsets based on the expression of CD45RA and CCR7 surface markers, as described by the groups of Sallusto and Lanzavecchia (Figure 5A). Representative distributions of CD8 T cells in blood and endocervix of $C$. trachomatis-negative and C. trachomatis-positive women are shown in Figure 5B. Analyses of blood and endocervical CD8 T cell subtype percent- distribution from 16 women using repeated ANOVA indicated a significant tissue site interaction $(\mathrm{p}<0.0001$; with Greenhouse-Geisser correction). Further, a paired comparison of mean percentage CD8 $\mathrm{T}$ cell subtypes in blood and endocervix, using $\mathrm{t}$ - test with confirmatory analysis using the Wilcoxon Signed Rank test, indicated a significantly higher percentage of $\mathrm{T}_{\mathrm{EM}}$ cells in the endocervix $(\mathrm{p}<0.0001)$, with a significantly lower percentage of naïve cells $(p<0.0001)$. Taken together these data indicate that, while the different CD8 $\mathrm{T}$ cell subtypes are relatively distributed in the blood, the endocervix is primarily populated by effector memory $\left(\mathrm{T}_{\mathrm{EM}}\right) \mathrm{CD} 8 \mathrm{~T}$ cells (Figure 5C and 5D).

\section{Effector memory CD8 $T$ cells ( $T_{E M S}$ ) in the human endocervix have limited perforin expression}

Based on the observation that the endocervix is primarily populated by CD8 $\mathrm{T}_{\mathrm{EM}}$ cells, we focused our subsequent analyses on this subpopulation. Here, the cytolytic potential of CD8 $T_{E M}$ cells was assessed by analyzing flow cytometry data for the percentage of perforin expressing CD8 $\mathrm{T}_{\mathrm{EM}}$ cells, using difference scores (blood - endocervix) and non-parametric analysis. Based on the Wilcoxon Rank Sum test, a significant difference in the percentage of perforin-expressing CD8 $\mathrm{T}_{\mathrm{EM}}$ cells from blood and endocervix were observed in both $C$. trachomatis-negative $(\mathrm{p}<0.05)$ and in $C$. trachomatis-positive $(\mathrm{p}<0.0001)$ women as shown in Figures 6A and $6 \mathrm{C}$ respectively. Furthermore, pair-wise comparison of the percentage of perforin $+T_{E M}$ in the blood and endocervix of individual samples 


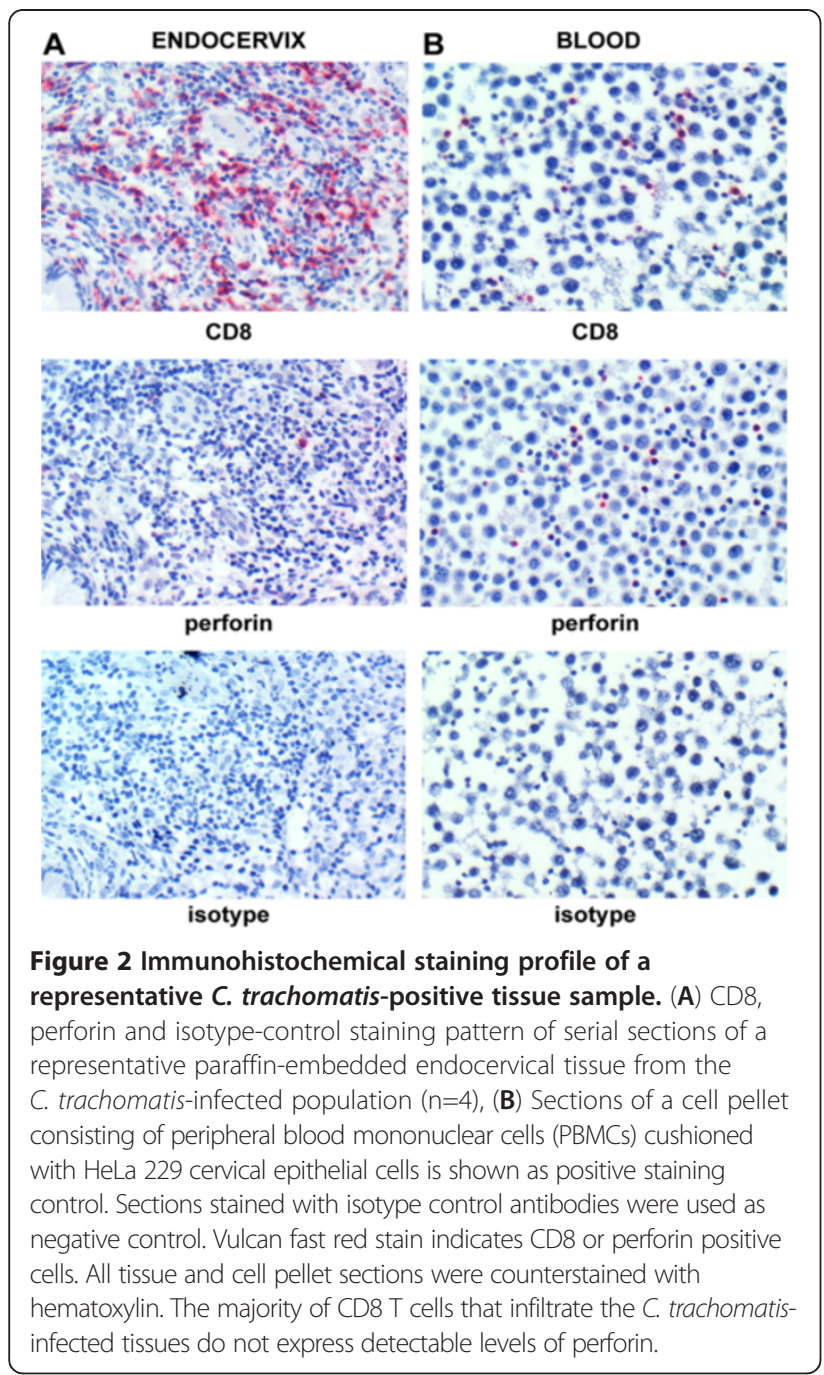

demonstrated that in both $C$. trachomatis-negative (Figure 6B) and $C$. trachomatis-positive patients (Figure 6D) there was a general trend for the endocervical $\mathrm{T}_{\mathrm{EM}}$ to have lower percentage of perforin positive cells compared to $\mathrm{T}_{\mathrm{EM}}$ derived from the blood. However, further analyses, with repeated measures ANOVA, using $C$. trachomatis infection status as a grouping factor, showed that although there were differences in blood vs. endocervix, no interaction with $C$. trachomatis-infection status was observed. These data show that despite the significant increase in CD8 T cells infiltrating the endocervix during C. trachomatis-infection, the relative low perforin level of the CD8 $\mathrm{T} \mathrm{T}_{\mathrm{EM}}$ cell population is still observed.

IFN gamma exposure of epithelial cells could potentially mediate the downmodulation of perforin expression of $\mathrm{T}_{\mathrm{EM}} \mathrm{CD} 8 \mathrm{~T}$ cells in the cervical microenvironment Our ex vivo data suggested that endocervical CD8 T $\mathrm{T}_{\mathrm{EM}}$ cells have a low perforin content. Therefore, we investigated one of the factors that could influence this phenotype. We hypothesized that IFN gamma could be one of the mediating factors that drives the decrease in perforin content of endocervical CD8 $\mathrm{T}_{\mathrm{EM}}$ cells based on the following observations from previous studies: 1) IFN gamma levels in the female genital tract are elevated during the secretory stage of menstruation [16] and during decidualization in successful pregnancies [17]; 2) Animal models of $C$. trachomatis infections have demonstrated that $\mathrm{T}$ cells with the capacity to secrete IFN gamma migrate to the site of Chlamydia infection [18]; 3) High levels of IFN gamma are found in the FGT of C. trachomatis-infected patients [19]; and 4) IFN gamma can induce the expression of indoleamine-2,3dioxygenase (IDO) in epithelial cells [20]; and IDO, a tryptophan catabolic enzyme, could downmodulate perforin expression in CD8 T cells [21]. Therefore, based on these previous findings, we developed a PBL-HeLa 229 cervical epithelial cell co-culture model to investigate whether the exposure of epithelial cells to IFN gamma has an effect on perforin expression by CD8 $\mathrm{T}_{\mathrm{EM}}$ cells. To do this, PBLs were cultured alone or with a monolayer of HeLa 229 cells and with or without $600 \mathrm{U} / \mathrm{mL}$ of IFN gamma (Figure 7A). After 38 hours in culture, the PBLs were then stained to identify the different CD8 $\mathrm{T}$ cell subpopulations and intracellular perforin and granzyme expression. The gating strategy to delineate the different CD8 $\mathrm{T}$ cell subpopulation is shown in Figure 7B. This gating allowed us to identify the CD8 $\mathrm{T}_{\mathrm{EM}}$ cell subpopulation from the PBLs, and to assess the perforin expression of these cells in the absence or presence of IFN gamma.

We found that the level of perforin expression by the control CD8 $\mathrm{T}_{\mathrm{EM}}$ cells from PBLs cultured in the absence of HeLa 229 cells did not shift in the presence of IFN gamma (Figure 7C - left panel). However, when we cocultured PBLs with HeLa cells, the addition of IFN gamma in the culture medium resulted in the negative shift in the fluorescence intensity of perforin, suggesting a downmodulation of the expression of this protein (Figure 7C - right panel). Therefore, our in vitro data suggest that IFN gamma could be potential mediator of the downmodulation of perforin expression in CD8 $\mathrm{T}_{\mathrm{EM}}$ cells.

\section{Discussion}

Using both in situ tissue-based and ex vivo cytobrushsampling protocols we confirm here that CD8 T cells comprise a significant component of the endocervical $\mathrm{T}$ cell infiltrate during C. trachomatis infection. Moreover, we also report that endocervical CD8 T cells are primarily of the $\mathrm{T}_{\mathrm{EM}}$ subtype, but, unlike their peripheral counterparts that express high levels of perforin, these cells have low levels of this protein. Since low perforin content is observed in CD8 $\mathrm{T}_{\mathrm{EM}}$ cells from both normal and C. trachomatis-infected tissues we hypothesize that the 


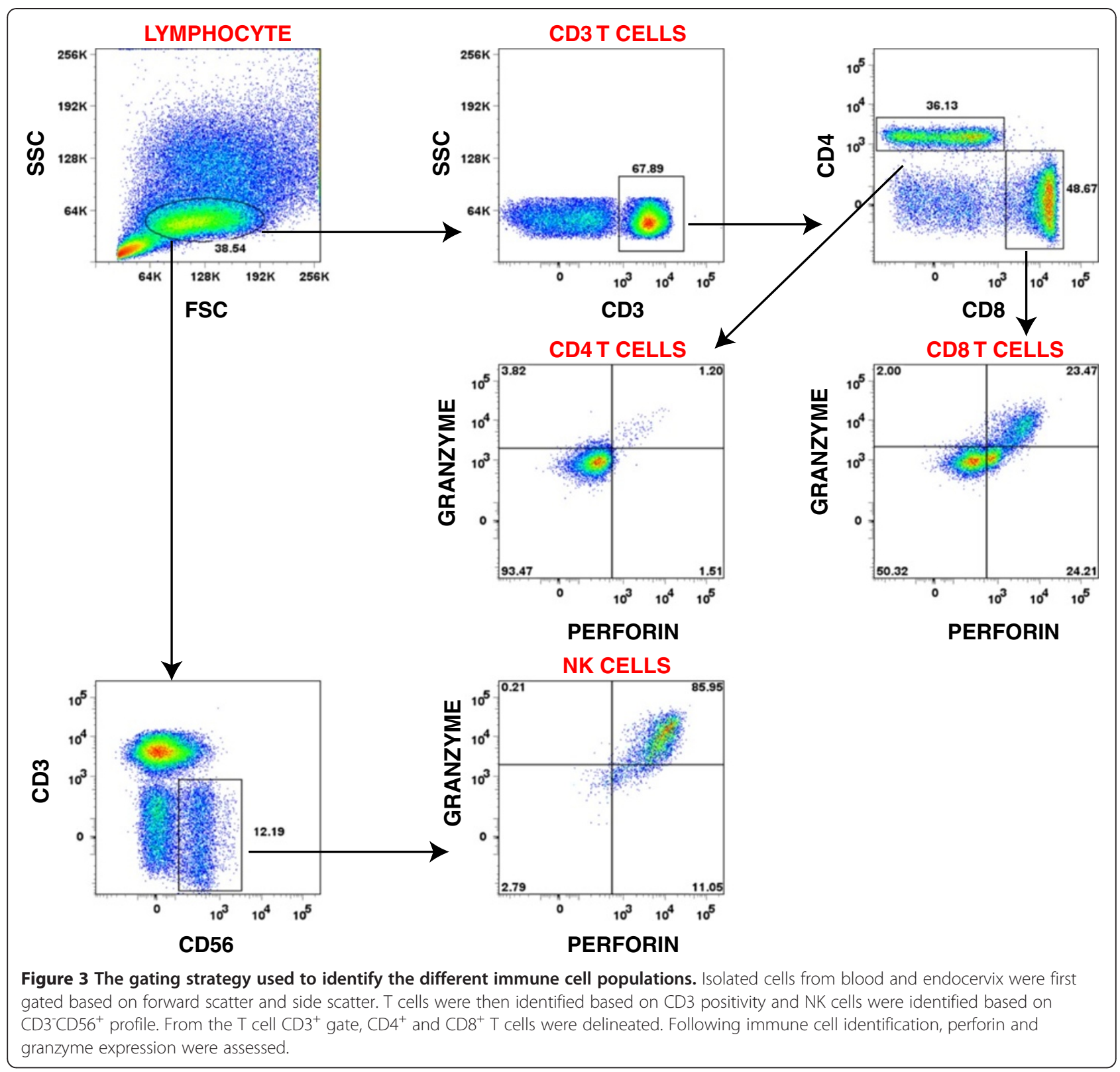

endocervical milieu drives this phenotype. That the endocervical CD8 $\mathrm{T}_{\mathrm{EM}}$ subpopulation is low in perforin may reflect an immunological response pattern that supports feto-maternal tolerance and facilitates a fine balance between protection of tissue integrity and defence against sexually transmitted pathogens.

Human studies and animal models of $C$. trachomatis infection have previously demonstrated recruitment of immune cells to the local site of infection. [18,22-29]. Compelling evidence for the ability of Chlamydiaspecific CD8 T cells to migrate to infected murine FGT has been presented by Roan and Starnbach who generated retrogenic mice expressing a $\mathrm{T}$ cell receptor specific for CrpA, a Chlamydia specific T cell antigen [18]. They observed that adoptively-transferred Chlamydia-specific retrogenic CD8 $\mathrm{T}$ cells proliferated in genital tractdraining lymph nodes of naïve recipients in response to genital $C$. trachomatis challenge. Further, they also demonstrated that the adoptively transferred Chlamydiaspecific CD8 $\mathrm{T}$ cells successfully migrated to the genital mucosa and acquired the ability to produce IFN gamma [ibid]. In our earlier study, in which we observed elevated endocervical $\mathrm{T}$ cell numbers during culture positive endocervical C. trachomatis infections, we also noted that $\mathrm{T}$ cells numbers significantly decline in these women post successful antibiotic treatment [30]. We interpret these observations to suggest that active $C$. trachomatis infection is most likely responsible for the recruitment of $\mathrm{T}$ 


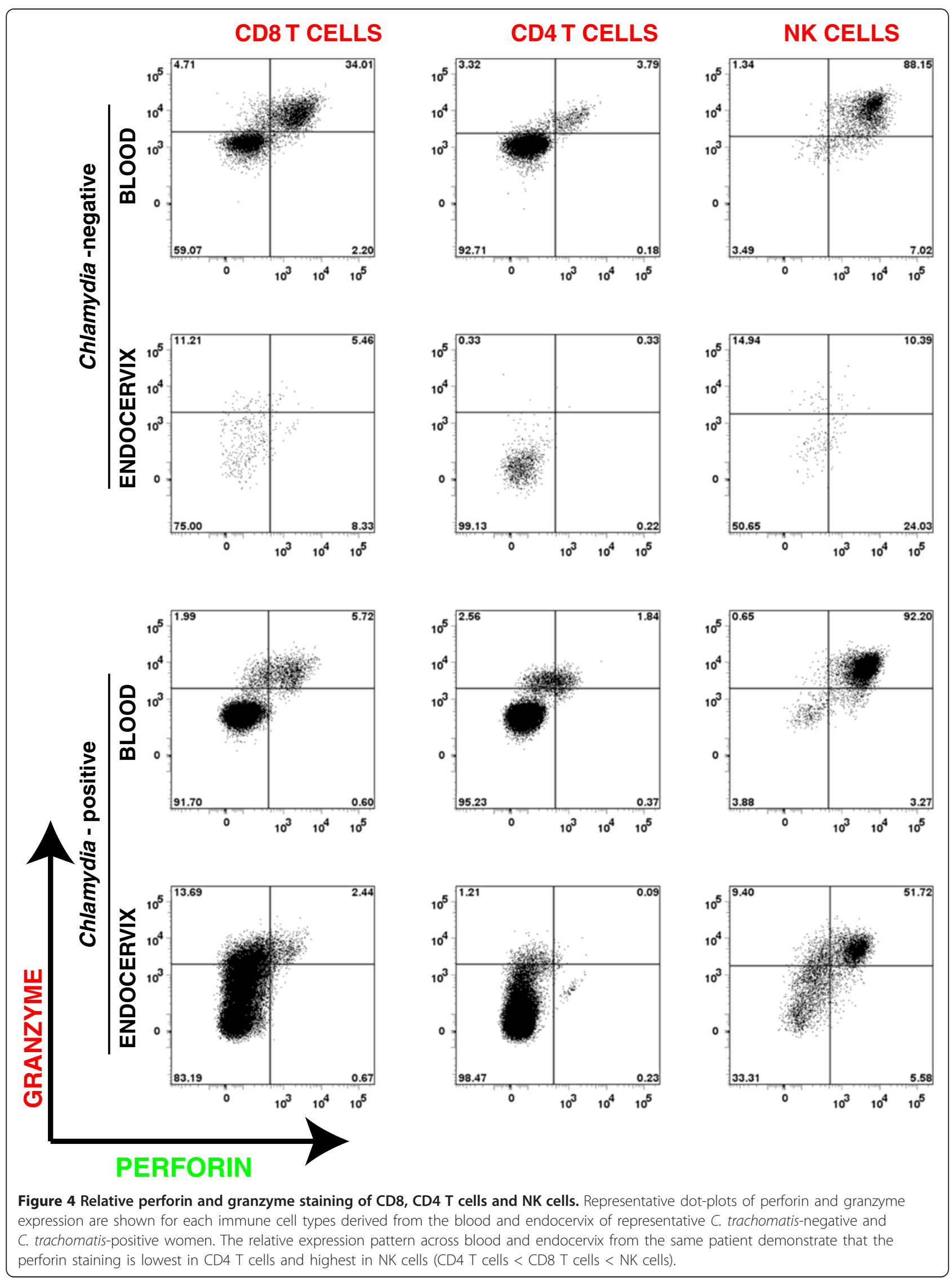




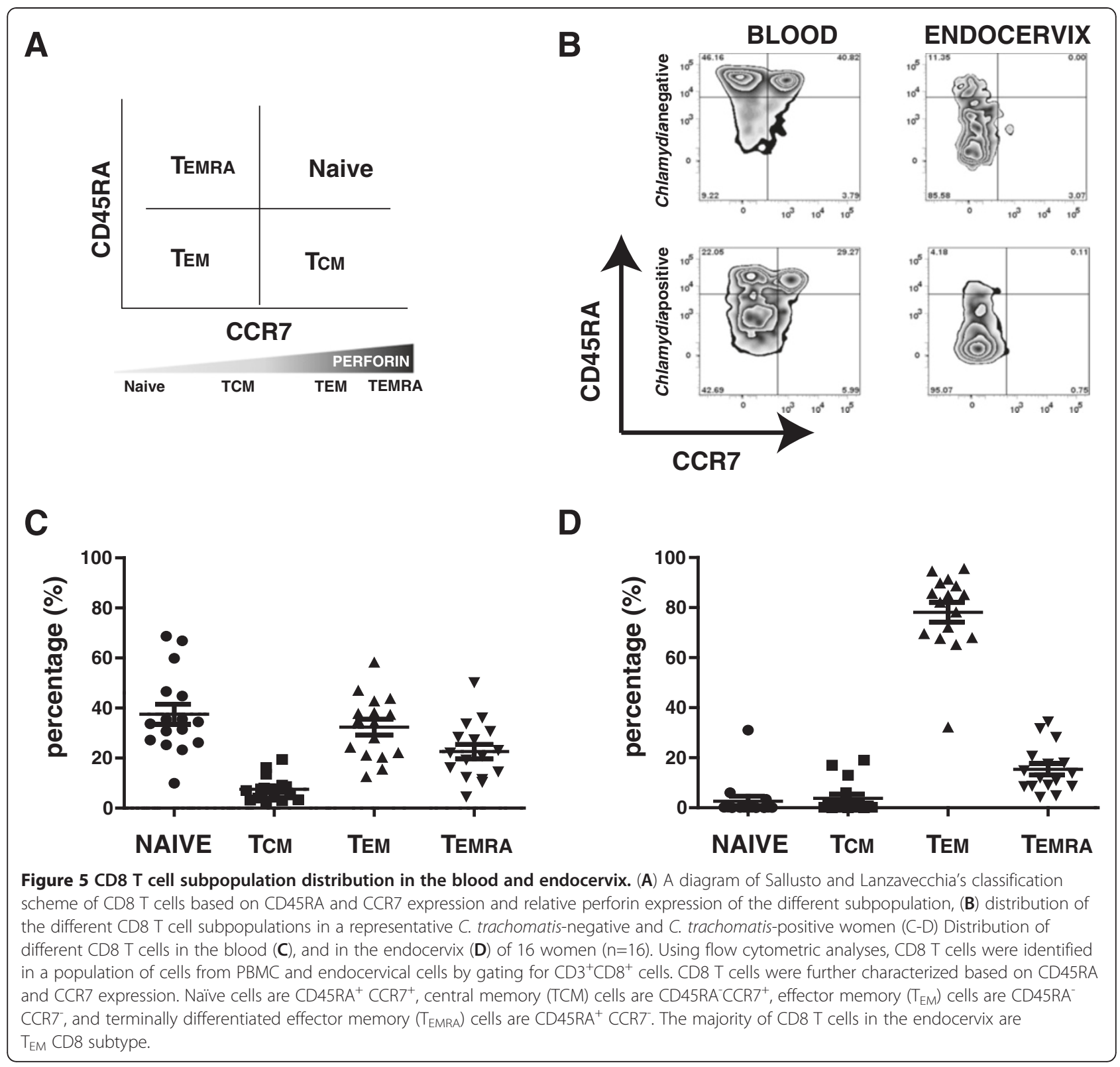

cells at the site of infection. We also observed that CD8 T cells constitute a significant proportion of the $\mathrm{T}$ cell infiltrates found in the endocervix [ibid]. Thus, our data from human study, and reports based on animal studies mutually support the finding that CD8 T cells migrate into the FGT in response to $C$. trachomatis infection.

Intriguingly, although animal and human studies suggest that CD8 $\mathrm{T}$ cells are recruited to the site of C. trachomatis infection, the role of their cytolytic activity in protective immunity remains uncertain. Some studies suggest that CD8 $\mathrm{T}$ cells can play a role in clearance of C. trachomatis infection [26,31], while other studies implicate these cells to be responsible for immunemediated pathology. As an example, Voorhis et al. found an association between perforin positive CD8 T cells and salpingeal tissue scarring in non-human primate model of C. trachomatis infection [32]. Further, in a study by Murthy et al., CD8 T cells were suggested to mediate oviduct pathology (hydrosalpinx) following C. muridarum infection in mice, but this was via their TNF $\alpha$ activity. This finding was demonstrated by the restoration of hydrosalpinx upon repletion of mice genetically deficient in CD8 T cells with perforin-deficient, but not with TNF $\alpha$-deficient, CD8 T cells [33]. Therefore, currently, there is no direct evidence that perforin plays a role in immune-mediated pathology by CD8 T cells during $C$. trachomatis infection. Furthermore, evidence based on lung and genital infection studies in perforin-knockout 

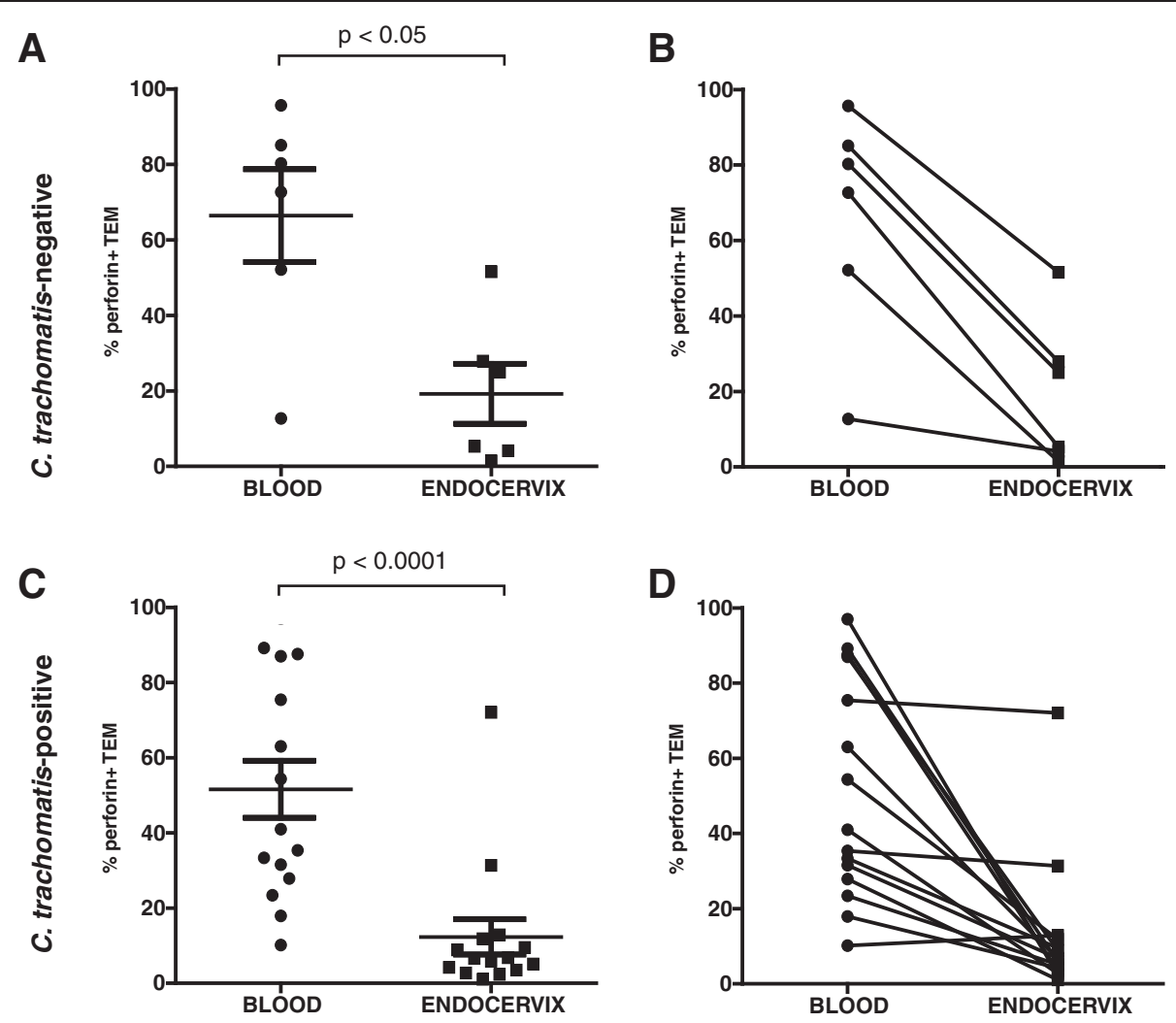

Figure 6 Effector memory CD8 (TEM) cells in the endocervix are low in perforin. (A) \%perforin+ CD45RA CCR7 CD8 ${ }^{+} T$ cells $T_{E M}$ in the blood and endocervix of $C$. trachomatis-negative $(n=6)$ women, $(\mathbf{B})$ a comparison of perforin expression in paired blood and endocervical derived $T_{E M}$ C. trachomatis-negative women, (C) \%perforin+ of $T_{E M}$ in the blood and endocervix of $C$. trachomatis-positive $(n=15)$ women, (D) a comparison of perforin expression in paired blood and endocervical derived TEM C. trachomatis-positive women. Statistical analyses were performed using difference scores (blood - endocervix) and non-parametric Wilcoxon Rank Sum test.

mice suggests that perforin is not required for clearance of murine chlamydial infection [34]. Based on these animal models, we could surmise that the perforindependent CD8 $\mathrm{T}$ cell cytolytic response may not play a critical role in controlling C. trachomatis burden in the human endocervix.

While studies by others and us suggest a limited role for perforin-dependent CD8 $\mathrm{T}$ cell cytolytic activity against $C$. trachomatis, we do not discount the possibility that CD8 $\mathrm{T}$ cells may be involved in perforinindependent immune mechanisms. Particularly, we believe that during $C$. trachomatis infection in the human endocervix, CD8 $\mathrm{T}$ cells may significantly contribute to the accumulation of IFN gamma at this site as demonstrated in murine studies by Starnbach's group [18,31]. This finding is supported by a report based on a human study that IFN gamma levels are elevated in the endocervix of C. trachomatis-infected women compared to uninfected controls [19].

Two of the possible mechanisms that could induce low perforin content in CD8 $\mathrm{T}_{\mathrm{EM}}$ in Chlamydia-infected endocervix are discussed here. These are: 1) CD8 $\mathrm{T}_{\mathrm{EM}}$ cells that have migrated to the endocervix in response to C. trachomatis infection exhibit active and continuous degranulation resulting in the loss of their perforin content, and; 2) The physiologic pressure in the female genital tract drives perforin downmodulation in CD8 $\mathrm{T}_{\mathrm{EM}}$ cells. To our knowledge, the differences in degranulation sensitivity and the rates of granule protein recovery between CD8 T cells and NK cells are not yet well defined. However, we note here that unlike the CD8 $\mathrm{T}_{\mathrm{EM}}$ cells, we observed that endocervical NK cells have a high perforin content (representative data shown in Figure 4), suggesting that our observations are not due to spontaneous and continuous degranulation of all cytolytic immune cells. Further, although not the focus of this study, we believe that our data suggests that NK cells could maintain a high cytolytic potential in the endocervix, and are likely to be a key immune cell population that mediates host cytolytic immune response to $C$. trachomatis infection. Consistent to this notion is our previous finding that the NK cell ligand expression in C. trachomatis infected endocervical epithelial cells renders these cells more susceptible to NK cell-mediated cytolysis [35]. 


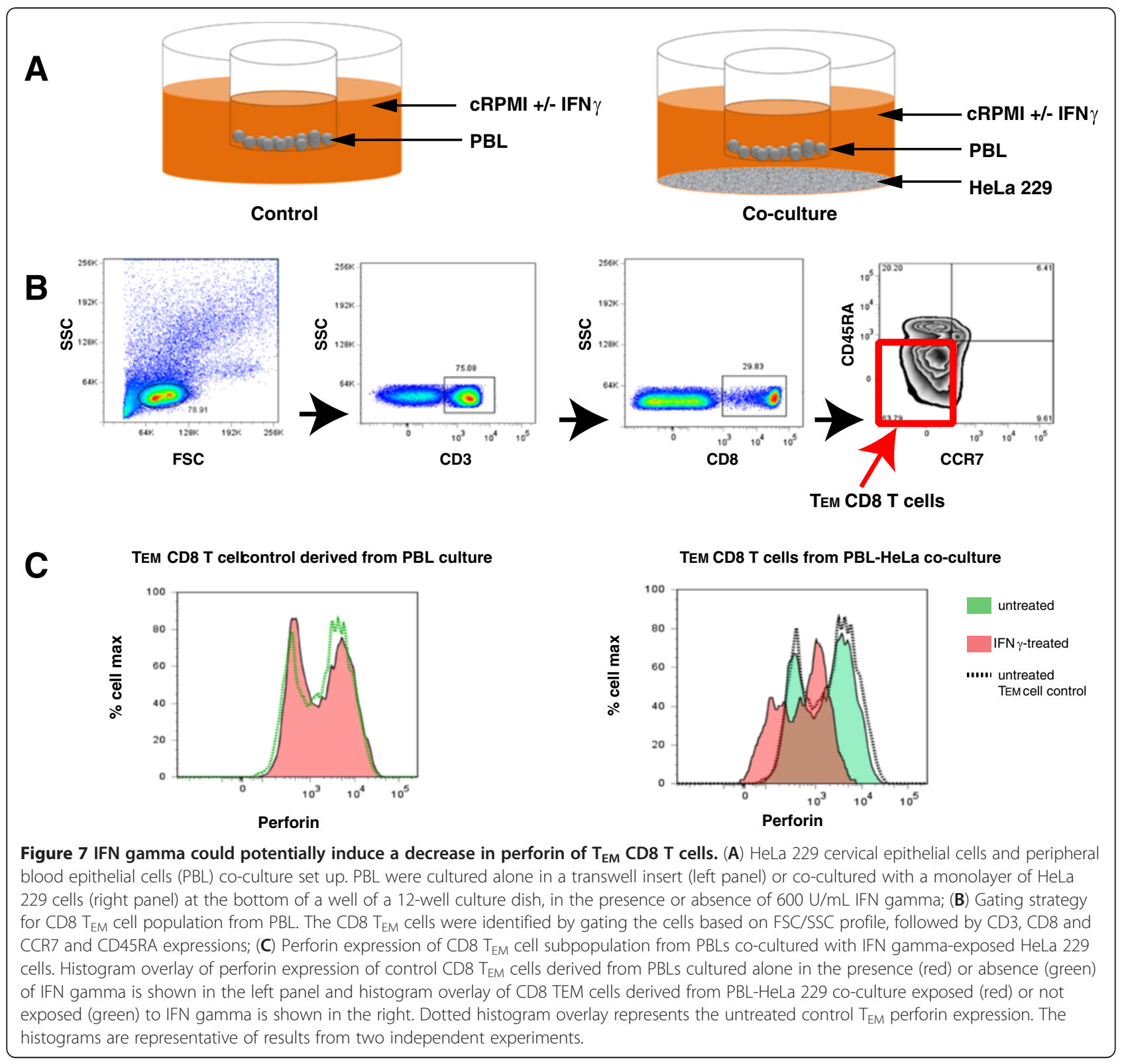

To address the first possibility, a study on degranulation status of CD8 $\mathrm{T}$ cell infiltrates in the endocervix would need to be undertaken. One experimental approach to test this possibility would be the concurrent measurement of the surface expression of CD107a, a marker of degranulation [36], and the intracellular perforin content of CD8 T cells derived from the endocervix. In principle, the low perforin content of CD8 T cells in the endocervix of women could result from their degranulation in response to stimulation of resident Chlamydia-specific, and other pathogen - specific CD8 T cells at this site. As we have not tested for degranulation in this study, we do not discount that this is a plausible mechanism that could underlie the low perforin content of CD8 $\mathrm{T}$ cells in the endocervix. Therefore, further study needs to be undertaken to test this possibility.

The second possibility, that physiological pressure in the endocervix drives the low perforin content of CD8 T cells, was explored in this study. Specifically, we have provided a proof-of-principle that the IFN gamma exposure of cervical epithelial cells could potentially downmodulate perforin expression of CD8 $\mathrm{T}_{\mathrm{EM}}$ cells in the same microenvironment. IFN gamma has been well established to induce indoleamine-2,3-dioxygenase (IDO) expression in epithelial cells. We also confirmed this in the HeLa 229 cells that were utilized in our in vitro study by immunoblotting HeLa 229 lysates with an IDO1-specific antibody (data not shown). IDO has 
been reported to impair the cytotoxic function of CD8 T cells by reducing perforin expression in these cells [21]. Therefore, IFN gamma secretion by infiltrating CD8 T cells during $C$. trachomatis infection may lead to an increase in the levels of IDO in the endocervix that could induce the decrease in perforin content of the local CD8 $\mathrm{T}_{\mathrm{EM}}$ cells. While we have not yet been able to test this hypothesis in vivo, we believe that further investigation into IDO and perforin regulation in the endocervix during $C$. trachomatis infection is warranted to determine the mechanisms and functional significance of the low perforin content of CD8 $\mathrm{T}_{\mathrm{EM}}$ cells in this unique anatomical region.

Interestingly, there are indications that our hypothesis, proposing that IFN gamma and IDO could mediate the downmodulation perforin expression of endocervical CD8 T cells during C. trachomatis infection, could be generally operant in the FGT even in the absence of chlamydial infection. The first series of studies that support our hypothesis includes the observation that endometrial tissues have high levels of IFN gamma at the secretory stage of the menstrual cycle but not during the proliferative phase [16]. Consistent with these findings are immunohistochemical and mRNA-based analyses demonstrating that IDO expression is low during the proliferative stage, but is elevated during the secretory phase $[37,38]$. Significantly, an elegant study by Wira's group demonstrated that $\mathrm{T}$ cells isolated from tissues during proliferative phase of menstrual cycle demonstrate cytolytic capacity but those during the secretory phase do not [39]. Therefore, the presence of IFN gamma and elevated levels of IDO expression during secretory phase, which can downmodulate perforin expression in CD8 $\mathrm{T}$ cells, coincides with the dampening of cytolytic $\mathrm{T}$ cell activity.

The second series of studies that support our hypothesis is based on the analyses of IDO expression during pregnancy. Elevated IDO expression has been found in the epithelium of cervical glands, Fallopian tubes and endometrial stromal cells during decidualization in both animal and human studies [37,38,40,41]. Interestingly, CD8 $\mathrm{T}$ cells comprise the largest fraction of $\mathrm{T}$ cells at the fetal-maternal interface [42]. However, analysis of perforin expression of CD8 $\mathrm{T}$ cells during pregnancy revealed that while peripheral CD8 $\mathrm{T}$ cells express perforin, this cytolytic molecule is deficient in decidual CD8 T cells $[43,44]$. These finding is consistent with the studies by Mellor et al. suggesting that IDOmediated $\mathrm{T}$ cell dysfunction plays a significant role in the induction of feto-maternal tolerance [45-48]. Therefore, it seems apparent that the IFN gamma-IDOperforin axis is an important component of the regulation of CD8 $\mathrm{T}$ cell cytolytic activity in the female genital tract.
If our hypothesis that IFN gamma-mediated induction of IDO is the primary mechanism involved in lowering perforin content of CD8 $\mathrm{T}_{\mathrm{EM}}$ cells in the endocervix, studies on the CD8 $\mathrm{T}$ cell immune repertoire of C. trachomatis infected women stratified based on their stage of menstrual cycle at the time of sample collection would be desirable, as this would clarify whether elevation of IFN gamma levels during $C$. trachomatis infection could overcome the low IDO expression during the proliferative stage. This would shed light on whether regardless of the stage of the menstrual cycle, the CD8 $\mathrm{T}_{\mathrm{EM}}$ cells in the endocervix are consistently low in perforin content during C. trachomatis infection.

\section{Conclusions}

The findings in this study suggest that perforindependent CD8 $\mathrm{T}$ cell-mediated cytoxicity may play a limited role in immune control of human endocervical C. trachomatis infection. However, further studies are needed to clearly establish the mechanism that drives the decrease in perforin levels in normal and C. trachomatis-infected endocervix. Nevertheless, despite the challenging nature of human studies in the female genital tract, we have generated ex vivo data that provides important insights into the endocervical immunological repertoire that may influence the outcome of C. trachomatis infection at this site.

\section{Methods}

Immunohistochemical assessment of CD8 T cell infiltrates Archived endocervical tissue paraffin blocks were utilized to assess the perforin and granzyme expression of endocervical CD8 $\mathrm{T}$ cell infiltrates in situ. Collection of endocervical tissues was approved by Louisiana State University Health Sciences Center Institutional Review Board. The endocervical tissues were derived from biopsies or discarded hysterectomy specimen. All specimens were screened for the presence of inclusions by chlamydial LPS staining. A confirmatory test of chlamydial positivity was performed on 3 of the 4 chlamydial-LPS positive tissues by ompA genotyping as previously described [30]. Endocervical tissues positive for C. trachomatis infection $(\mathrm{n}=4)$ and endocervical tissues negative for C. trachomatis infection $(n=6)$ were analyzed by immunohistochemical staining for CD3, CD8 and perforin expression. In each experiment, sections of cell pellet consisting of peripheral blood mononuclear cells buffered with HeLa 229 cells were used as a positive control, while staining with isotype-matched irrelevant antibodies were used as negative controls. Immunohistochemical staining was undertaken by first cutting $4 \mu \mathrm{M}$ sections of tissue which were immediately fixed on glass slides. Paraffin wax was then removed by baking the slides at $65^{\circ} \mathrm{C}$ for 1 hour, after which the slides were 
soaked in Antigen Retrieval solution (Biocare) and heated in a de-cloaking chamber (Biocare) for $20 \mathrm{~min}$ utes. The processed sections of tissues on the slides were then blocked using Background Sniper blocking reagent (Biocare) to prevent non-specific background staining for 1 hour at room temperature. Individual tissue sections were then reacted with the following primary antibodies: Anti-CD3 (Dakocytomation), anti-CD8 (Biocare), anti-perforin (Biolegend) or isotype-matched control (BD Biosciences) solution and incubated for 1 hour. After several washes with phosphate buffered saline (PBS), the tissue sections were incubated with appropriate secondary-antibodies conjugated to alkaline phosphatase. The slides were then washed with PBS to remove unbound antibodies, and Vulcan Fast Red chromogen system (Biocare) was added and allowed to develop. After a substrate reaction was observed in positive controls, the slides were soaked in distilled water and counterstained with hematoxylin. Tissues were then dehydrated in a series of methanol and fixed on the slide by covering with coverslip with permanent mounting medium, VectaMount (Vector Laboratories). The differences in number of CD3 and CD8 T cell infiltrates were assessed using Student's t-test after checking the assumptions of normality and homogeneity of variance, followed by confirmatory analyses with Wilcoxon Rank Sum test.

\section{Patient population}

To assess of the cytolytic potential of CD8 T cells in the blood and endocervix, we recruited participants into a study through the Delgado Clinic, New Orleans, Louisiana. Written informed consent as approved by the Louisiana State University Health Sciences Center Institutional Review Board was obtained from each participant. A total of 21 paired blood and endocervical cell samples were collected from women 18-30 years old attending the clinic who were enrolled based on satisfying at least one of the following criteria: (1) clinical evidence of cervicitis, (2) having a male partner who was tested positive for $C$. trachomatis infection $(\mathrm{CT}+)$ and (3) a positive test result for $C$. trachomatis infection by nucleic acid amplification test (NAAT) 3 to 30 days prior to enrolment. The participants provided information on demographics, history of previous sexually transmitted infections, antibiotic usage, and sexual behaviour. The participants were also examined by a nurse practitioner for mucupurulent cervical and vaginal discharge, bleeding and presence of clue cells. Urine samples were obtained to test for Neisseria gonorrhoea and C. trachomatis infection by NAAT. The $C$. trachomatis positive population were defined by a positive $C$. trachomatis NAAT result and confirmed with a C. trachomatis positive culture. Additional vaginal swabs were taken to assess for Trichomonas vaginalis, yeast infection, and bacterial vaginosis as previously described [15]. HIV positive patients were excluded from the study.

\section{Sample collection and processing}

To analyze the immune cell repertoire in the endocervix of women enrolled in the study, parallel samples of whole blood $(40 \mathrm{~mL})$ and endocervical cells collected using two cytobrushes were performed as previously described. Peripheral blood mononuclear cells (PBMC) were isolated by conventional ficoll differential centrifugation, and endocervical cells were isolated from the cytobrushes following the previously established protocol in our laboratory [30].

\section{Immunophenotyping by multiparameter flow cytometry}

To analyze the cytolytic potential of systemic and endocervical CD8 T cells, polychromatic (6-color) flow cytometry was performed. After processing the PBMC and endocervical cells, aliquots of these cells were made. One aliquot of cells was stained with fluorescently labeled antibodies against surface markers: CD56-APC (BD Pharmingen), CD3-PerCP Cy5.5 (BD Biosciences), CD4-Alexa Fluor 700 (Biolegend), CD8-APC-Cy7 (BD Pharmingen). Second aliquot for Isotype controls was stained with isotype-matched fluorescent antibodies: APC IgG1k Isotype (BD Biosciences), PerCP Cy5.5 IgGk isotype (BD Biosciences), Alexa Flour 700 IgG1k isotype (Biolegend), APC-Cy7 IgG1k Isotype (BD Biosciences). The cells were then permeabilized using Cytofix/Cytoperm (BD Pharmingen), a formulation of paraformaldehyde and saponin, to fix and permeabilize the cells. Following permeabilization, the cells were stained for intracellular proteins using labeled antibodies against Perforin-FITC (Abcam) and Granzyme B-PE (Cell Sciences). The second isotype aliquot was stained with FITC IgG2b Isotype (BD Biosciences) and PE IgG1 Isotype (BD Biosciences). CD4 T cells and NK cells were also analyzed to serve as cellular controls. Granzyme was utilized as intracellular staining control. Using the polychromatic flow cytometry approach, the different immune cells were simultaneously identified and cytolytic potentials measured. The percentage of cells positive for perforin was determined by gating the cells based on the threshold fluorescence of the FITC IgG2b Isotype control.

\section{CD8 T cell phenotyping}

To further analyze CD8 $\mathrm{T}$ cell subpopulations, a third aliquot of cell preparation were stained for CD3-PerCP Cy5.5 and CD8-APC Cy7 in conjunction with CCR7APC (R\&D Systems) and CD45 RA-Alexa Fluor 700 (AbD Serotec), perforin-FITC (Abcam) and granzyme BPE (CellSciences). This is to categorize the different 
subpopulations of CD8 $\mathrm{T}$ cells following the classification by Sallusto et al. [8,9]. Further phenotyping of CD8 $\mathrm{T}$ cells allowed the identification of the different CD8 T cell subpopulation for a more extensive analysis of memory CD8 $\mathrm{T}$ cell population.

\section{PBL-HeLa 229 cervical epithelial cell co-culture}

HeLa 229 cervical epithelial cells were obtained from the ATCC, and cultured in complete RPMI 1640 (cRPMI) culture medium with $10 \%$ human AB serum (Sigma); supplemented with $200 \mathrm{mM}$ glutamine (Invitrogen) and $0.25 \%(\mathrm{wt} / \mathrm{vol})$ glucose (Sigma). HeLa cells were seeded in a 12-well culture plate and cultivated for 24 to 36 hours until about $80 \%$ confluence was achieved. Peripheral blood mononuclear cells (PBMCs) were isolated from whole blood as described above. The isolated PBMCs were then placed in 6-well culture dish and incubated at $5 \% \mathrm{CO} 2$ at $37^{\circ} \mathrm{C}$ for 4 hours to allow monocytes to adhere to the plastic. The non-adherent-PBLs were then collected, washed and counted using a heamocytometer. The PBLs were then placed on cell culture transwell insert on laid on top of a well on a 12 well plate without or with HeLa cells. The cells were cultured for 38 hours in the absence or presence of $600 \mathrm{U} / \mathrm{mL}$ IFN gamma in cRPMI. The cells were harvested, and the viability of the cells were assessed by trypan blue staining to confirm that $\sim 95 \%$ of the cells are viable. The cells were then stained with monoclonal antibodies against CD3, CD8, CD45RA, CCR7, perforin and granzyme, and flow cytometric analyses were performed as described above.

\section{Statistical analyses}

Data were summarized using means (+ standard deviations), medians, and percentages as appropriate. Normality assumptions were assessed using the Shapiro-Wilks test, and where they were violated, parametric analyses were confirmed with analogous nonparametric methods. Differences between levels of CD3 and CD8 cells in C. trachomatis-positive and C. trachomatis-negative tissues were tested using Student's t-test (Wilcoxon Rank Sum test). Paired blood and endocervical specimens were assessed usi ng paired t-test (Wilcoxon Signed Rank test), and repeated measures ANOVA (Signed Rank and Rank Sum tests) was used to determine if blood versus endocervical differences varied with $C$. trachomatis infection status (interaction). SAS statistical software (version 9.2) was used for all analyses.

\section{Competing interests}

The authors declare that they have no competing interests.

\section{Authors' contributions}

JAl: performed all the assays, analyzed data, and wrote the manuscript. LM: participated in the design of the study and carried out the statistical analyses. CP: participated in flow cytometric data acquisition and analyses.
ML: participated in immunohistochemical data acquisition and analyses. ST: participated in the design of the study and collection of samples. DHM: participated in the design and coordination of the study, helped to write the manuscript. AJQ: conceived the study, participated in the design and coordination and helped to write the manuscript. All authors read and approved the final manuscript.

\section{Acknowledgements}

This work is dedicated to Ms. Barbara Smith, RNP, who, until her untimely passing, worked tirelessly not only in collecting samples but in caring for patients attending the Delgado Clinic. The study was funded by NIH grant U19AI061972 and the LSUHSC Research Enhancement Fund. Flow cytometric experiments were performed at the LSUHSC Flow Cytometry Shared Resource that is supported in part as a shared facility of the LSUHSC School of Medicine, the Comprehensive Alcohol Research Center (NIAAA P60AA009803) and by the Louisiana Vaccine Center (Louisiana Board of Regents).

\section{Author details}

${ }^{1}$ Microbiology, Immunology and Parasitology Department, Louisiana State University Health Sciences Center, New Orleans, Louisiana 70112, USA.

${ }^{2}$ Section of Pulmonary/Critical Care, Department of Medicine, Louisiana State University Health Sciences Center, New Orleans, Louisiana 70112, USA.

${ }^{3}$ School of Public Health and Tropical Medicine, Tulane University, New Orleans, Louisiana 70112, USA. ${ }^{4}$ Section of Infectious Diseases, Department of Medicine, Louisiana State University Health Sciences Center, New Orleans, Louisiana 70112, USA.

Received: 11 July 2012 Accepted: 3 December 2012

Published: 7 December 2012

\section{References}

1. Smyth MJ, Trapani JA: Granzymes: exogenous proteinases that induce target cell apoptosis. Immunol Today 1995, 16(4):202-206.

2. Trapani JA, Jans DA, Jans PJ, Smyth MJ, Browne KA, Sutton VR: Efficient nuclear targeting of granzyme $B$ and the nuclear consequences of apoptosis induced by granzyme B and perforin are caspase-dependent, but cell death is caspase-independent. J Biol Chem 1998, 273(43):27934-27938.

3. Uellner R, Zvelebil MJ, Hopkins J, Jones J, MacDougall LK, Morgan BP, Podack E, Waterfield MD, Griffiths GM: Perforin is activated by a proteolytic cleavage during biosynthesis which reveals a phospholipidbinding C2 domain. EMBO J 1997, 16(24):7287-7296.

4. Tschopp J, Schafer S, Masson D, Peitsch MC, Heusser C: Phosphorylcholine acts as a Ca2+-dependent receptor molecule for lymphocyte perforin. Nature 1989, 337(6204):272-274.

5. Kagi D, Ledermann B, Burki K, Seiler P, Odermatt B, Olsen KJ, Podack ER, Zinkernagel RM, Hengartner $\mathrm{H}$ : Cytotoxicity mediated by $\mathrm{T}$ cells and natural killer cells is greatly impaired in perforin-deficient mice. Nature 1994, 369(6475):31-37.

6. Lanzavecchia A, Sallusto F: Understanding the generation and function of memory T cell subsets. Curr Opin Immunol 2005, 17(3):326-332.

7. Germain RN: MHC-dependent antigen processing and peptide presentation: providing ligands for T lymphocyte activation. Cell 1994, 76(2):287-299.

8. Sallusto F, Lenig D, Forster R, Lipp M, Lanzavecchia A: Two subsets of memory $T$ lymphocytes with distinct homing potentials and effector functions. Nature 1999, 401(6754):708-712.

9. Sallusto F, Geginat J, Lanzavecchia A: Central memory and effector memory T cell subsets: function, generation, and maintenance. Annu Rev Immunol 2004, 22:745-763.

10. Champagne P, Dumont AR, Sekaly RP: Learning to remember: generation and maintenance of T-cell memory. DNA Cell Biol 2001, 20(12):745-760

11. Sutton VR, Waterhouse NJ, Baran K, Browne K, Voskoboinik I, Trapani JA: Measuring cell death mediated by cytotoxic lymphocytes or their granule effector molecules. Methods 2008, 44(3):241-249.

12. Masopust D, Vezys V, Wherry EJ, Barber DL, Ahmed R: Cutting edge: gut microenvironment promotes differentiation of a unique memory CD8 T cell population. J Immunol 2006, 176(4):2079-2083.

13. Shacklett BL, Cox CA, Quigley MF, Kreis C, Stollman NH, Jacobson MA, Andersson J, Sandberg JK, Nixon DF: Abundant expression of granzyme A, 
but not perforin, in granules of CD8+ T cells in GALT: implications for immune control of HIV-1 infection. J Immunol 2004, 173(1):641-648.

14. Rukavina D, Laskarin G, Rubesa G, Strbo N, Bedenicki I, Manestar D, Glavas M, Christmas SE, Podack ER: Age-related decline of perforin expression in human cytotoxic T lymphocytes and natural killer cells. Blood 1998, 92(7):2410-2420.

15. Arnold V, Balkow S, Staats R, Matthys H, Luttmann W, Virchow JC Jr: Increase in perforin-positive peripheral blood lymphocytes in extrinsic and intrinsic asthma. Am J Respir Crit Care Med 2000, 161(1):182-186.

16. Kumar S, Li Q, Dua A, Ying YK, Bagchi MK, Bagchi IC: Messenger ribonucleic acid encoding interferon-inducible guanylate binding protein 1 is induced in human endometrium within the putative window of implantation. J Clin Endocrinol Metab 2001, 86(6):2420-2427.

17. Vives A, Balasch J, Yague J, Quinto L, Ordi J, Vanrell JA: Type-1 and type-2 cytokines in human decidual tissue and trophoblasts from normal and abnormal pregnancies detected by reverse transcriptase polymerase chain reaction (RT-PCR). Am J Reprod Immunol 1999, 42(6):361-368.

18. Roan NR, Starnbach MN: Antigen-specific CD8+ T cells respond to Chlamydia trachomatis in the genital mucosa. J Immunol 2006, 177:7974-7979.

19. Arno JN, Ricker VA, Batteiger BE, Katz BP, Caine VA, Jones RB: Interferongamma in endocervical secretions of women infected with Chlamydia trachomatis. J Infect Dis 1990, 162:1385-1389.

20. Feng GS, Taylor MW: Interferon gamma-resistant mutants are defective in the induction of indoleamine 2,3-dioxygenase. Proc Natl Acad Sci USA 1989, 86:7144-7148.

21. Liu H, Liu L, Bizargity P, Hancock WW, Visner GA: Reduced cytotoxic function of effector CD8+ T cells is responsible for indoleamine 2,3-dioxygenase-dependent immune suppression. J Immunol 2009, 183:1022-1031.

22. Olive AJ, Gondek DC, Starnbach MN: CXCR3 and CCR5 are both required for $\mathrm{T}$ cell-mediated protection against $\mathrm{C}$. trachomatis infection in the murine genital mucosa. Mucosal Immunol, 4(2):208-216.

23. Rank RG, Bowlin AK, Kelly KA: Characterization of lymphocyte response in the female genital tract during ascending Chlamydial genital infection in the guinea pig model. Infect Immun 2000, 68(9):5293-5298.

24. Morrison SG, Morrison RP: In situ analysis of the evolution of the primary immune response in murine Chlamydia trachomatis genital tract infection. Infect Immun 2000, 68(5):2870-2879.

25. Starnbach MN, Bevan MJ, Lampe MF: Murine cytotoxic T lymphocytes induced following Chlamydia trachomatis intraperitoneal or genital tract infection respond to cells infected with multiple serovars. Infect Immun 1995, 63(9):3527-3530.

26. Starnbach MN, Bevan MJ, Lampe MF: Protective cytotoxic T lymphocytes are induced during murine infection with Chlamydia trachomatis. J Immunol 1994, 153(11):5183-5189

27. Kelly KA, Wiley D, Wiesmeier E, Briskin M, Butch A, Darville T: The combination of the gastrointestinal integrin (alpha4beta7) and selectin ligand enhances T-Cell migration to the reproductive tract during infection with Chlamydia trachomatis. Am J Reprod Immunol 2009, 61(6):446-452

28. Kelly KA, Rank RG: Identification of homing receptors that mediate the recruitment of CD4 T cells to the genital tract following intravaginal infection with Chlamydia trachomatis. Infect Immun 1997, 65(12):5198-5208.

29. Hawkins RA, Rank RG, Kelly KA: Expression of mucosal homing receptor alpha4beta7 is associated with enhanced migration to the Chlamydiainfected murine genital mucosa in vivo. Infect Immun 2000, 68(10):5587-5594.

30. Ficarra M, Ibana JS, Poretta C, Ma L, Myers L, Taylor SN, Greene S, Smith B, Hagensee $M$, Martin DH, et al: A distinct cellular profile is seen in the human endocervix during Chlamydia trachomatis infection. Am J Reprod Immunol 2008, 60(5):415-425.

31. Lampe MF, Wilson CB, Bevan MJ, Starnbach MN: Gamma interferon production by cytotoxic $\mathrm{T}$ lymphocytes is required for resolution of Chlamydia trachomatis infection. Infect Immun 1998, 66(11):5457-5461.

32. Van Voorhis WC, Barrett LK, Sweeney YT, Kuo CC, Patton DL: Repeated Chlamydia trachomatis infection of Macaca nemestrina fallopian tubes produces a Th-1-like cytokine response associated with fibrosis and scarring. Infect Immun 1997, 65:2175-2182.
33. Murthy AK, Li W, Chaganty BK, Kamalakaran S, Guentzel MN, Seshu J, Forsthuber TG, Zhong G, Arulanandam BP: Tumor necrosis factor alpha production from CD8+ T cells mediates oviduct pathological sequelae following primary genital Chlamydia muridarum infection. Infect Immun, 79(7):2928-2935.

34. Perry LL, Feilzer K, Hughes S, Caldwell HD: Clearance of Chlamydia trachomatis from the murine genital mucosa does not require perforin-mediated cytolysis or Fas-mediated apoptosis. Infect Immun 1999, 67(3):1379-1385.

35. Ibana JA, Aiyar A, Quayle AJ, Schust DJ: Modulation of MICA on the surface of Chlamydia trachomatis-infected endocervical epithelial cells promotes NK cell-mediated killing. FEMS Immunol Med Microbiol, 65(1):32-42.

36. Betts MR, Brenchley JM, Price DA, De Rosa SC, Douek DC, Roederer M, Koup RA: Sensitive and viable identification of antigen-specific CD8+ T cells by a flow cytometric assay for degranulation. J Immunol Methods 2003, 281(1-2):65-78

37. Sedlmayr P, Blaschitz A, Wintersteiger R, Semlitsch M, Hammer A, MacKenzie CR, Walcher W, Reich O, Takikawa O, Dohr G: Localization of indoleamine 2,3-dioxygenase in human female reproductive organs and the placenta. Mol Hum Reprod 2002, 8(4):385-391.

38. Kudo Y, Hara T, Katsuki T, Toyofuku A, Katsura Y, Takikawa O, Fujii T, Ohama $\mathrm{K}$ : Mechanisms regulating the expression of indoleamine 2,3dioxygenase during decidualization of human endometrium. Hum Reprod 2004, 19(5):1222-1230.

39. White HD, Crassi KM, Givan AL, Stern JE, Gonzalez JL, Memoli VA, Green WR, Wira CR: CD3+ CD8+ CTL activity within the human female reproductive tract: influence of stage of the menstrual cycle and menopause. J Immunol 1997, 158(6):3017-3027.

40. Drenzek JG, Breburda EE, Burleigh DW, Bondarenko Gl, Grendell RL, Golos TG: Expression of indoleamine 2,3-dioxygenase in the rhesus monkey and common marmoset. J Reprod Immunol 2008, 78(2):125-133.

41. von Rango U, Krusche CA, Beier HM, Classen-Linke I: Indoleaminedioxygenase is expressed in human decidua at the time maternal tolerance is established. J Reprod Immunol 2007, 74(1-2):34-45.

42. Tilburgs T, van der Mast BJ, Nagtzaam NM, Roelen DL, Scherjon SA, Claas $\mathrm{FH}$ : Expression of NK cell receptors on decidual T cells in human pregnancy. J Reprod Immunol 2009, 80(1-2):22-32.

43. Tilburgs T, Scherjon SA, Roelen DL, Claas FH: Decidual CD8+CD28- T cells express CD103 but not perforin. Hum Immunol 2009, 70(2):96-100.

44. Tilburgs T, Schonkeren D, Eikmans M, Nagtzaam NM, Datema G, Swings GM, Prins F, van Lith JM, van der Mast BJ, Roelen DL, et al: Human decidual tissue contains differentiated CD8+ effector-memory T cells with unique properties. J Immunol, 185(7):4470-4477.

45. Munn DH, Zhou M, Attwood JT, Bondarev I, Conway SJ, Marshall B, Brown C, Mellor AL: Prevention of allogeneic fetal rejection by tryptophan catabolism. Science 1998, 281(5380):1191-1193.

46. Mellor AL, Sivakumar J, Chandler P, Smith K, Molina H, Mao D, Munn DH: Prevention of T cell-driven complement activation and inflammation by tryptophan catabolism during pregnancy. Nat Immunol 2001, 2(1):64-68.

47. Mellor AL, Munn DH: Extinguishing maternal immune responses during pregnancy: implications for immunosuppression. Semin Immunol 2001, 13(4):213-218.

48. Mellor AL, Munn DH: Tryptophan catabolism prevents maternal T cells from activating lethal anti-fetal immune responses. J Reprod Immunol 2001, 52(1-2):5-13.

\section{doi:10.1186/1471-2172-13-66}

Cite this article as: Ibana et al: The major CD8 T cell effector memory subset in the normal and Chlamydia trachomatis-infected human endocervix is low in perforin. BMC Immunology 2012 13:66. 